\title{
How to estimate the resolution of an LES of recirculating flow
}

\author{
Lars Davidson ${ }^{1}$ \\ Division of Fluid Dynamics, Department of Applied Mechanics, Chalmers \\ University of Technology, SE-412 96 Göteborg, Sweden lada@chalmers.se, \\ www.tfd.chalmers.se $/ \sim$ lada
}

\begin{abstract}
Summary. How should the resolution of an LES be estimated? There exist guidelines for boundary layers for how to create a grid in terms of the grid size expressed in viscous units. However, in other flow regions there are few - if any - guidelines for how to generate a grid that ensures accurate results. Worse, it is not even clear how to estimate the resolution after having carried out an LES. The present study evaluates the following quantities: energy spectra, dissipation energy spectra, twopoint correlations, the ratio of SGS shear stress to resolved shear stress, the ratio of the SGS viscosity to the molecular and the ratio of the SGS dissipation due to the resolved fluctuating velocity gradients to that due to the mean velocity gradients. Two flows are analyzed, namely the flow in a plane asymmetric diffuser and decaying grid turbulence. The main conclusions are that two-point correlations are the best way to estimate the resolution and that energy spectra are not suitable. It is usually assumed that the SGS dissipation takes place at wavenumbers close to cut-off. The present work shows that this idealized picture is not true, but that the SGS dissipation takes place at rather low wavenumbers.
\end{abstract}

Key words: LES, resolution, two-point correlations, energy spectra, dissipation energy spectra, decaying grid turbulence

\section{Introduction}

After having carried out a Large Eddy Simulation, the question arises: how do we know that the resolution is sufficient? Or - at least - how do we estimate the resolution? For attached boundary layer flows, numerical experiments reported in the literature indicate that the streamwise and spanwise resolution in viscous units should be approximately 100 and 30, respectively; further, the center of the wall-adjacent cells should be located not more than one viscous unit away from the wall. However, in flow regions outside attached boundary layers, there are few guidelines for how to create a sufficiently fine mesh. [5] 
After having carried out an LES simulation, there are a number of ways to estimate the resolution. Energy spectra are frequently used to find out whether the resolved turbulence satisfies the $-5 / 3$ decay. The ratio of the SGS to resolved turbulence is another quantity; if is is small, it may indicate that the resolution is good. Two-point correlations are also useful for estimating the resolution. If they fall to zero over a separation distance of a few cells, it means that no turbulence is resolved at all; if, on the other hand, they fall to zero over five to ten cells, this is an indication that the largest scales are reasonably well resolved. Another option for evaluating the resolution is to evaluate the SGS dissipation. This dissipation takes place either through the mean flow or through the resolved fluctuations. In RANS, the entire dissipation takes place through the mean flow while in LES it takes places through both the mean flow and resolved fluctuations. Finally, dissipation energy spectra can be created that show the wavenumbers at which the SGS dissipation takes place.

The different ways of estimating resolution mentioned above were evaluated in [6] for fully developed channel flow. In the present study, we use these methods to evaluate the resolution of an LES of recirculating flow and of decaying grid turbulence.

\section{Equations}

\subsection{The momentum equations}

The incompressible momentum equation with an added SGS viscosity reads

$$
\frac{\partial \bar{u}_{i}}{\partial t}+\frac{\partial}{\partial x_{j}}\left(\bar{u}_{i} \bar{u}_{j}\right)=-\frac{1}{\rho} \frac{\partial \bar{p}}{\partial x_{i}}+\frac{\partial}{\partial x_{j}}\left(\left(\nu+\nu_{s g s}\right) \frac{\partial \bar{u}_{i}}{\partial x_{j}}\right)
$$

\subsection{The turbulence model}

The dynamic Smagorinsky is used, which reads

$$
\nu_{s g s}=C \Delta^{2}|\bar{s}|, \quad \bar{s}_{i j}=\frac{1}{2}\left(\frac{\partial \bar{u}_{i}}{\partial x_{j}}+\frac{\partial \bar{u}_{j}}{\partial x_{i}}\right), \quad|\bar{s}|=\left(2 \bar{s}_{i j} \bar{s}_{i j}\right)^{1 / 2}
$$

The dynamic coefficient is computed as

$$
\begin{aligned}
C & =-\frac{\left\langle\mathcal{L}_{i j} M_{i j}\right\rangle_{z}}{\left\langle 2 M_{i j} M_{i j}\right\rangle_{z}} \\
M_{i j} & =\left(\widehat{\Delta}^{2}|\widehat{s}| \widehat{s}_{i j}-\Delta^{2} \overparen{|\bar{s}| \bar{s}_{i j}}\right), \quad \mathcal{L}_{i j} \equiv{\overparen{\bar{u}_{i} \bar{u}_{j}}}-\widehat{\bar{u}}_{i} \widehat{\bar{u}}_{j}
\end{aligned}
$$

where $\frown$ and $\langle.\rangle_{z}$ denote test filtering and spanwise averaging, respectively. The dynamic coefficient is limited to avoid negative total viscosity, i.e. $\nu+$ $\nu_{s g s} \geq 0$. 


\subsection{The Numerical Method}

An incompressible, finite volume code is used [7]. For space discretization, central differencing is used for all terms in the momentum equations. The Crank-Nicolson scheme (with $\alpha=0.5$ ) is used. Convective boundary conditions are used at the outlet. The numerical procedure is based on an implicit, fractional step technique with a multigrid pressure Poisson solver [8] and a non-staggered grid arrangement.

\subsection{Inlet boundary conditions}

A fluctuating velocity field is generated each time step using a synthetic isotropic turbulence generator [4]. The velocity fields are independent of each other, however, and their time correlation will thus be zero. This is unphysical. To create correlation in time, new fluctuating velocity fields, $\mathcal{U}_{i}^{\prime}$, are computed based on an asymmetric time filter.

$$
\mathcal{U}_{i}^{\prime m}=a \mathcal{U}_{i}^{\prime m-1}+b u_{i}^{\prime m} .
$$

Here, $m$ denotes the time step number, and $a=\exp (-\Delta t / \mathcal{T}) ; \Delta t$ and $\mathcal{T}$ denote the computational time step and a prescribed turbulent time scale, respectively. The asymmetric time filter resembles the spatial digital filter presented in [10]. The second coefficient is taken as $b=\left(1-a^{2}\right)^{0.5}$ which ensures that $\left\langle\mathcal{U}_{i}^{\prime 2}\right\rangle=\left\langle u_{i}^{\prime 2}\right\rangle\left(\langle\cdot\rangle\right.$ denotes averaging). The time correlation of $\mathcal{U}_{i}^{\prime}$ will be equal to $\exp (-\Delta t / \mathcal{T})$, and thus Eq. 4 is a convenient way to prescribe the turbulent time scale of the fluctuations. The inlet boundary conditions are prescribed as

$$
\bar{u}_{i}(0, y, z, t)=U_{i, i n}(y)+\mathcal{U}_{i}^{\prime m}(y, z)
$$

$U_{i, i n}(y)$ denotes the mean inlet profile, which is taken from a DNS of fully developed channel flow at $R e_{\tau}=500$. For greater detail, see [4]

The turbulent length scale and time scale are set to $L_{t}=0.1 \mathrm{H} / 2$ and $\mathcal{T}=0.05(H / 2) / u_{\tau, \text { in }}$ (see Fig. 1), respectively. The RMS amplitudes of the inlet fluctuations are scaled so that $u_{r m s, i n}=v_{r m s, i n}=w_{r m s, i n}=u_{\tau}$

The synthetic fluctuations created with the method presented above yield homogeneous turbulence in the inlet plane, i.e. $u_{r m s, i n}, v_{r m s, i n}$, and $w_{r m s, i n}$ are constant (and equal) across the entire inlet plane. The fluctuations must be reduced near the wall so that they go smoothly to zero as the wall is approached; this is done for $n / H \lesssim 0.016$, where $n$ is the distance to the closest wall.

\section{Results}

\subsection{Diffuser}

The configuration is an asymmetric plane diffuser with Reynolds number $R e=U_{b, i n} H / \nu=18000$, see Fig. 1 . The opening angle is $10^{\circ}$. The inlet bulk 
velocity, the channel height at the inlet and the density are set to one, so that $\nu=1 / R e$. The dynamic Smagorinsky model is used, and predictions are compared with experiments [1]. The mesh in the $x-y$ plane has $256 \times 64\left(N_{x} \times N_{y}\right)$ or $512 \times 64$ cells (two cells in the $x$ direction in the latter mesh correspond to one cell in the former mesh). In the $z$ direction, $N_{z}=32,64$ or 128 . In the inlet region, the friction Reynolds number based on half the channel height is approximately 500, which gives a spanwise grid size of $\Delta z^{+}=(125,62,31)$ for $N_{z}=(32,64,128)$. The time step is $\Delta t=0.023$ for $N_{x}=512$ and $\Delta t=0.039$ for $N_{x}=256$; this results in a maximum instantaneous Courant number of 1.9. Time averaging is carried out over approximately 60000 time steps. All simulations are made on a Linux PC using a single core. One or two global iterations are required each time step to reach convergence. The CPU time for the finest mesh $(512 \times 64 \times 128)$ is $45 s$ per time step (two iterations per time step).

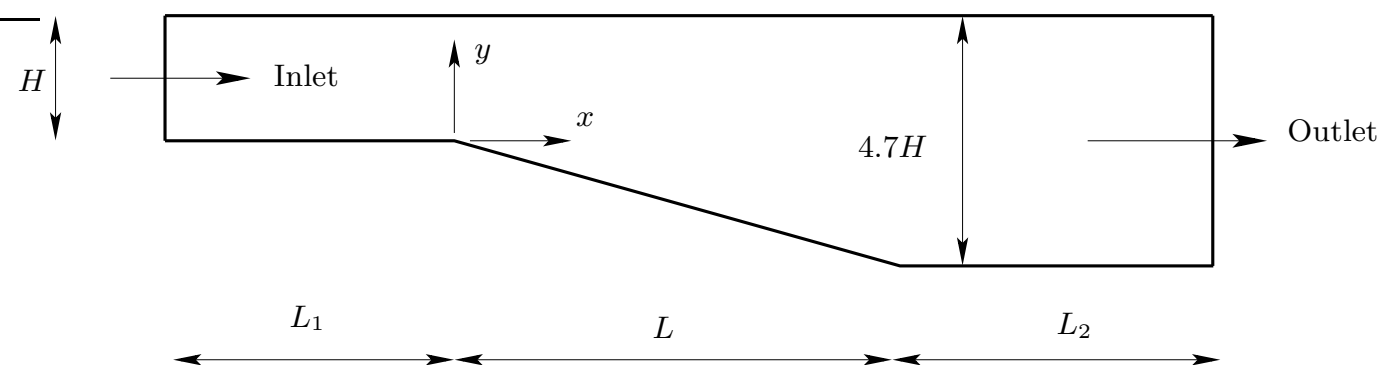

Fig. 1. Plane asymmetric diffuser (not to scale). $L_{1}=7.9 H, L=21 H, L_{2}=28 H$. The spanwise width is $z_{\max }=4 H$. The origin of $x-y-z$ is at the lower wall at the entrance of the diffuser.

Figure 2 compares the predicted velocity profiles with experiments [1]. The agreement is not especially good for any resolution. LES simulations were also presented in [12]. Their meshes were similar; they employed the same turbulence model and their domain was shorter but had the same spanwise extent. They did use a finer mesh in the wall-normal direction and different inlet boundary conditions. However, the aim of the present work is not to achieve as good an agreement with experiments as possible but to evaluate the resolution for different grids.

The resolved shear stresses are presented in Fig. 3. The agreement is reasonable for $x \geq 13 H$ but the stresses are overpredicted compared with experiments for $x \leq 6 H$. All resolutions give very similar shear stresses.

Below we will make a detailed comparison of the flow at one streamwise position where the flow is attached $(x=-H)$ and one where the flow exhibits incipient separation $(x=20 H)$. At $x=-H$ the ratio of streamwise to the spanwise cell side is 0.3 for $N_{z}=32$ and $N_{x}=512$; at $x=20 H$ the corresponding ratio is one. Figures 4 and 5 show the shear stresses. As can be seen, 


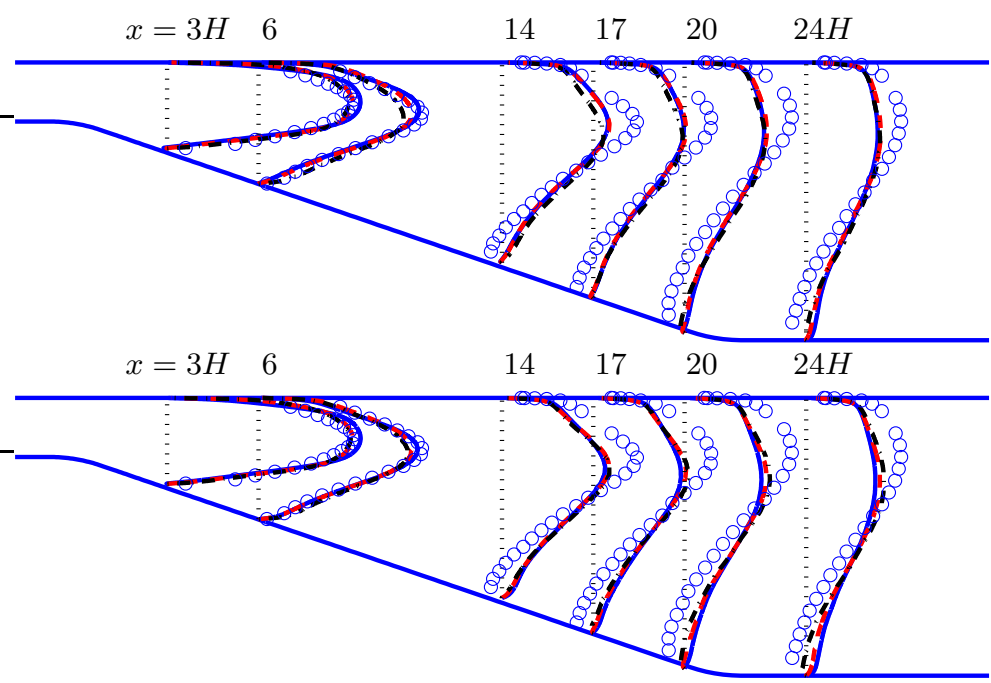

Fig. 2. $\langle\bar{u}\rangle / U_{b, \text { in }}$ profiles. Top: $N_{x}=256$ cells; bottom: $N_{x}=512$ cells. — $N_{z}=32 ;---: N_{z}=64 ;-.-: N_{z}=128$; markers: experiments [1].

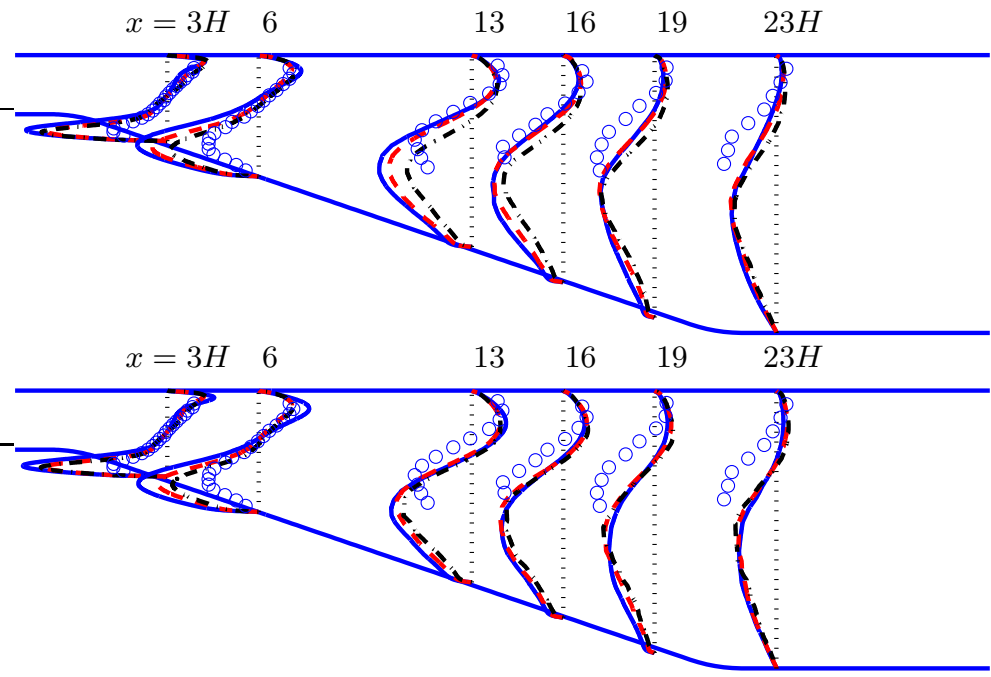

Fig. 3. $\left\langle u^{\prime} v^{\prime}\right\rangle / U_{b, i n}^{2}$ profiles. Top: $N_{x}=256$ cells; bottom: $N_{x}=512$ cells. For legend, see caption in Fig. 2. Markers: experiments [1].

the spanwise resolution is very important in the attached boundary layer, but the effect of the streamwise resolution is almost negligible. Also the spanwise resolution is unimportant in the incipient separation region, and all grids give virtually identical resolved shear stresses. Since it is seen that the streamwise 

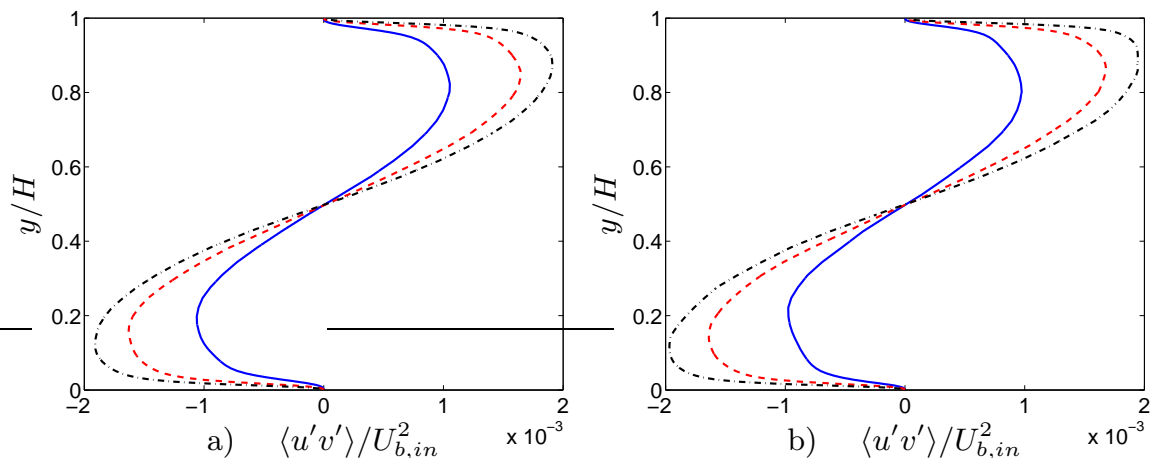

Fig. 4. $\left\langle u^{\prime} v^{\prime}\right\rangle / U_{b, i n}^{2}$ profiles. $x=-H$. Left: $N_{x}=256$ cells; right: $N_{x}=512$ cells. For legend, see caption in Fig. 2.

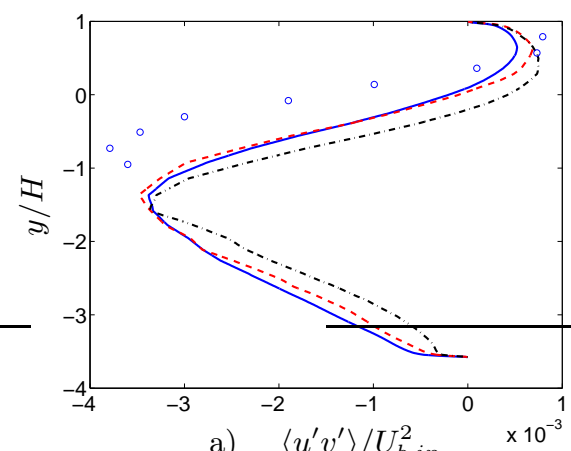

a) $\left\langle u^{\prime} v^{\prime}\right\rangle / U_{b, i n}^{2}$

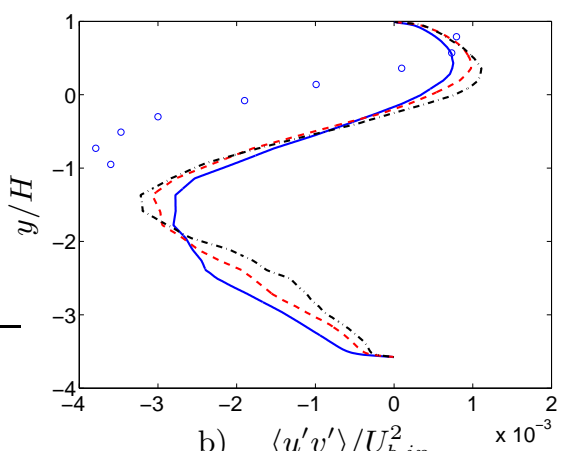

b) $\left\langle u^{\prime} v^{\prime}\right\rangle / U_{b, i n}^{2}$

Fig. 5. $\left\langle u^{\prime} v^{\prime}\right\rangle / U_{b, i n}^{2}$ profiles. $x=20 H$. Left: $N_{x}=256$ cells; right: $N_{x}=512$ cells. For legend, see caption in Fig. 2.

resolution has no effect, we concentrate hereafter on comparing the spanwise grid resolutions for the coarse streamwise grid $\left(N_{x}=256\right)$.

Figure 6 presents the two-point correlation $\left(B_{w w}(\hat{z})=\left\langle w^{\prime}(z) w^{\prime}(z-\hat{z})\right\rangle\right)$. The correlations are presented at $x=-H$ (attached flow) and $x=20 H$ (incipient separation) at $y$ locations for which the magnitude of the resolved shear stress is large (see Figs. 4 and 5). The resolution for the coarse grid $\left(N_{z}=32\right)$ is indicated by markers in Fig. 6 . As can be seen, the normalized two-point correlation at $x=-H$ (Fig. 6a) for $N_{z}=32$ falls to 0.1 within a separation distance of two cells. This means that the largest scales are resolved by only two cells, i.e. they are not resolved at all. For the medium mesh $\left(N_{z}=64\right)$ and the fine mesh $\left(N_{z}=128\right)$, the two-point correlations fall to 0.1 within four and eight cells, respectively. Clearly, both the coarse and medium meshes are too coarse. The resolution however is good in the separation region (Fig. 6b). Even in the case of the coarse grid, the largest scales are covered by some ten cells. 


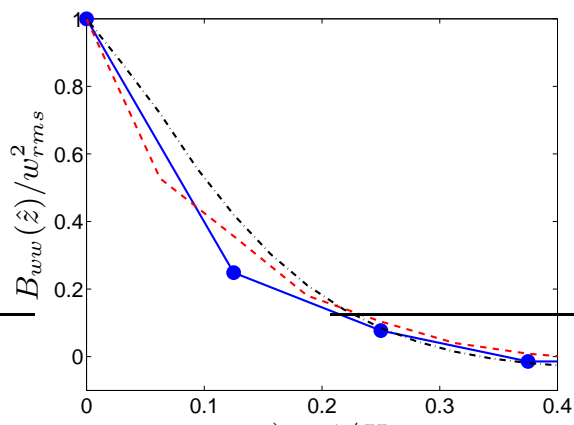

a) $\hat{z} / H$

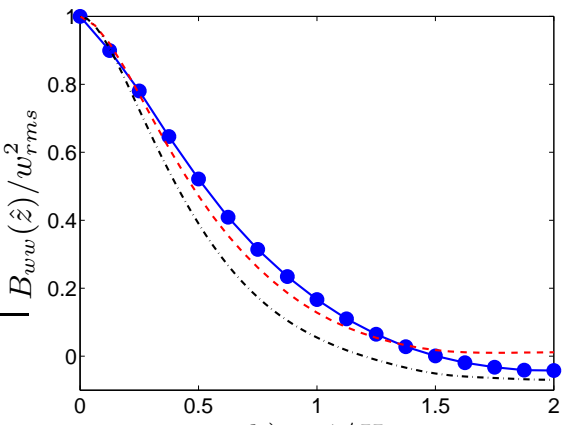

b) $\hat{z} / H$

Fig. 6. Normalized two-point correlation $B_{w w}(\hat{z}) / w_{r m s}^{2} . N_{x}=256$. Left: $x=-H$, $y=0.15 H$; right: $x=20 H, y=-2.9 H$. Markers on the solid line show the coarse resolution. For legend, see caption in Fig. 2.

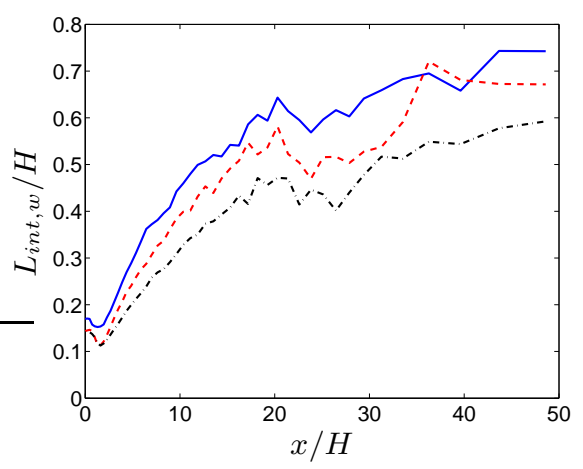

Fig. 7. Integral length scale, $L_{i n t, w}$ computed from $B_{w w}$, see Eq. 6. $(y-$ $\left.y_{\text {low }}\right) /\left(y_{\text {high }}-y_{\text {low }}\right)=0.15$. For legend, see caption in Fig. 2 .

The integral length scale, $L_{i n t, w}$, is computed from the two-point correlations as

$$
L_{i n t, w}(x, y, \hat{z})=\frac{1}{w(x, y)_{r m s}^{2}} \int_{0}^{z_{\text {limit }}}\left\langle w^{\prime}(x, y, z, t) w^{\prime}(x, y, z, t)\right\rangle d \hat{z}
$$

where the averaging $\langle$.$\rangle is done, as usual, in time (t)$ and the spanwise direction $(z)$. The upper boundary of the integral, $z_{\text {limit }}$, is $z_{\max }$ or when the normalized two-point correlation falls below a small value $\left(=10^{-4}\right)$. As can be seen in Fig. $7, L_{i n t, w}$ is reduced when the grid is refined. Figure 7 also confirms that the spanwise domain is sufficiently large, since $L_{i n t, w}<0.5 z_{\max }=2 \mathrm{H}$.

The energy spectra corresponding to the two-point correlations (Fig. 6) are shown in Fig. 8. The smallest wavenumber is $\kappa_{z, \min }=2 \pi / z_{\max }=2 \pi / 4 H=$ $1.57 / H$. The largest wavenumber must be resolved by more than two cells, which for $N_{z}=32$ gives $\kappa_{z, \max }<2 \pi /(2 \Delta z) \simeq 25 / H$. The decay of $E_{w w}$ with wavenumber is small for the coarse mesh. However, the energy spectra for 


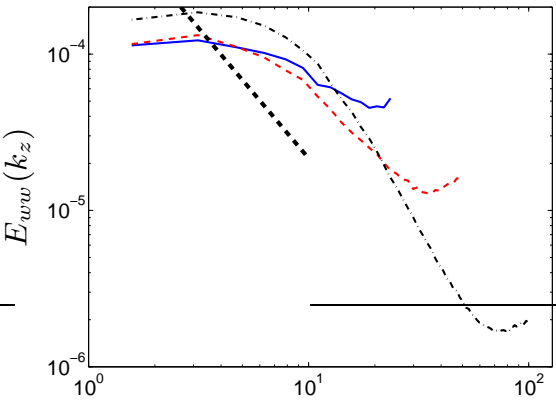

a) $\kappa_{z}=2 \pi\left(k_{z}-1\right) / z_{\max }$

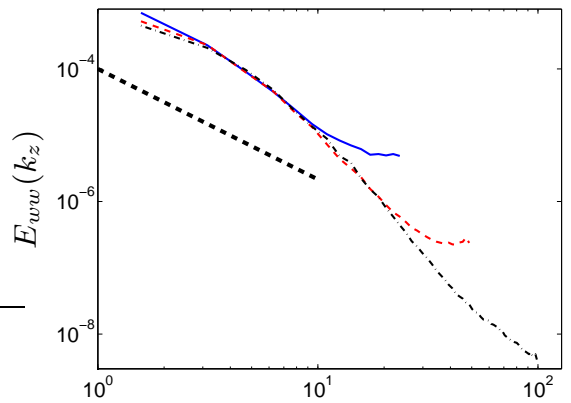

b) $\quad \kappa_{z}=2 \pi\left(k_{z}-1\right) / z_{\max }$

Fig. 8. Energy spectra $E_{w w}\left(k_{z}\right)$. The thick dashed line shows $-5 / 3$ slope. $N_{x}=256$. Left: $x=-H, y=0.15 H$; right: $x=20 H, y=-2.9 H$. For legend, see caption in Fig. 2.

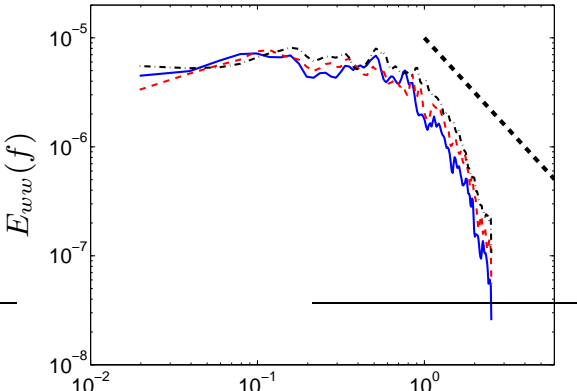

a) $\quad f$

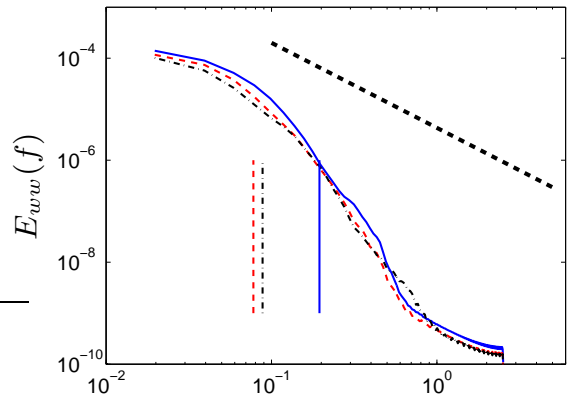

b) $f$

Fig. 9. Energy spectra $E_{w w}(f)$ vs. frequency. The thick dashed line shows $-5 / 3$ slope. $N_{x}=256$. Left: $x=-H, y=0.15 H$; right: $x=20 H, y=-2.9 H$. For legend, see caption in Fig. 2.

the medium and the fine mesh exhibit a decay versus wavenumber close to $-5 / 3$, indicating that the turbulence is well resolved. Still, as seen from the two-point correlation, the largest scales on the medium mesh are resolved by only four cells, which must be considered to be insufficient. Hence, it seems that energy spectra are not suitable for estimating the resolution. The energy spectra all exhibit a pile-up of energy at the largest wavenumber, and this indicates that the SGS dissipation is too small at these wavenumbers.

In the incipient separation region, Fig. 8b, all spectra show a decay slightly larger than $-5 / 3$, indicating a sufficient resolution. This indication agrees with the two-point correlations (Fig. 6b) for which it was seen that the largest scales are resolved by at least ten cells, even on the coarse mesh.

The energy spectra, $E_{w w}\left(\kappa_{z}\right)$, presented in Fig. 8 were obtained by Fourier transforming (FFT) the corresponding two-point correlation, $B_{w w}(\hat{z})$. This 
can be done only if $z$ is a homogeneous coordinate direction, i.e. if the predictions were carried out using periodic boundary conditions in the $z$ direction. This is of course not the case in general. The only way to create energy spectra is generally to Fourier transform the time history of a variable (e.g. a velocity component) at a given point. The time signal is usually chopped up into small segments, making an FFT of each segment using an overlap of the segments and then averaging the spectra of all segments. Special treatment is given here to make the signal in each segment periodic. Here, the pwelch command in Matlab is used setting the length of each segment to $N T=256$ (the time signal was created by sampling every fifth time step). The pwelch command reads

$$
[\mathrm{pw} 1 \mathrm{fw1}]=\operatorname{pwelch}(\mathrm{w} 1, \mathrm{NT},[],[], 1 /(5 * d t)) ;
$$

where $w 1, f w 1, p w 1$ and $d t$ denote the resolved spanwise velocity, $w^{\prime}$, the frequency, the square of the Fourier coefficients and the computational time step, respectively. The length of the w1 vector is approximately 10000 .

Figure 9 presents the spectra versus frequency. At $x=-H$, all spectra are flat for $f$ smaller than $1 H z$; for larger frequencies, they exhibit a $-5 / 3$ decay or steeper. At $x=20 H$, the spectra for the three resolutions are very similar and exhibit a rather steep decay for $f>0.1 \mathrm{~Hz}$. The steep decay is related to the fact that the time signal has been sampled with a frequency higher than that corresponding to a local Courant number of one. The vertical lines in Fig. 9b show the frequency based on the time averaged velocity over the spatial grid size, $\langle\bar{u}\rangle / \Delta x$. Frequencies above this threshold correspond to "over-resolution" in time, i.e. the local Courant number is smaller than one. In Fig. 9a the Courant number based on the sampling frequency (every fifth time step), is close to three, i.e. the frequency, $\langle\bar{u}\rangle / \Delta x$, is equal to approximately 14. The conclusion drawn from the spectra presented in Figs. 8 and 9 is that they do not present a reliable picture of the resolution. Considering Fig. 9a it seems that all three simulations are well resolved, and Fig. 8a indicates that the prediction with the medium mesh is well resolved. However, the two-point correlation (Fig. 6a) shows that it is only the simulation on the fine mesh $\left(N_{z}=128\right)$ that is reasonably well resolved.

The relation between modelled (SGS) and resolved turbulence can be used as an estimate of how well the turbulence is resolved. Large SGS stresses could indicate a poorly resolved simulation. Figure 10 presents the ratio of the SGS shear stress to the resolved one. As can be seen, the SGS shear stress is negligible in the incipient separation region while it reaches values of approximately $25 \%$ in the turbulent boundary layer at $x=-H$ for the coarse mesh.

Figure 11 shows the ratio of the turbulent viscosity to the physical one. For the coarse mesh, the ratio reaches a value of approximately 12 in the incipient separation region while it is three times smaller (approximately 4 ) in the attached flow region. Both ratios $\left\langle\tau_{s g s, 12}\right\rangle /\left\langle u^{\prime} v^{\prime}\right\rangle$ and $\left\langle\nu_{s g s}\right\rangle / \nu$ behave consistently: the better the resolution, the smaller they are. However, the ratio 


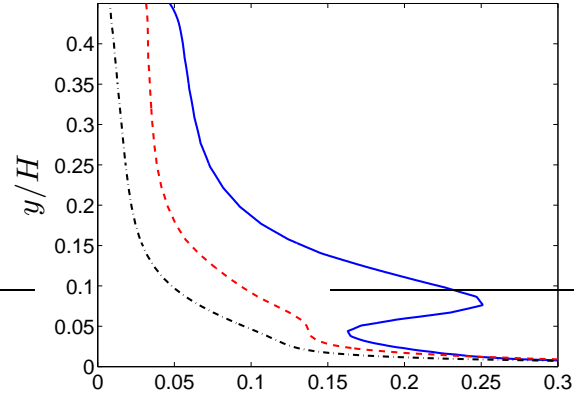

a) $\left\langle\tau_{s g s, 12}\right\rangle /\left\langle u^{\prime} v^{\prime}\right\rangle$

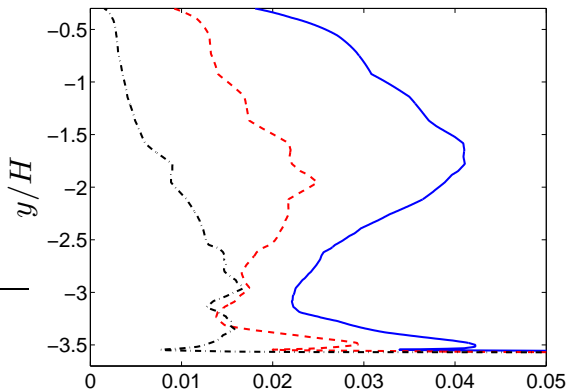

b) $\left\langle\tau_{s g s, 12}\right\rangle /\left\langle u^{\prime} v^{\prime}\right\rangle$

Fig. 10. Ratio of SGS to resolved Reynolds shear stress. $N_{x}=256$. Left: $x=-H$; right: $x=20 H$. For legend, see caption in Fig. 2 .

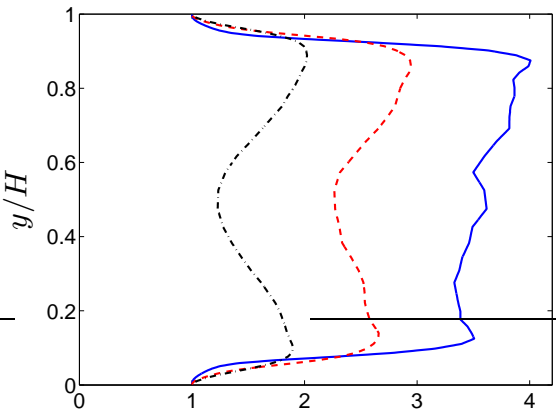

a) $\left\langle\nu_{s g s}\right\rangle / \nu$

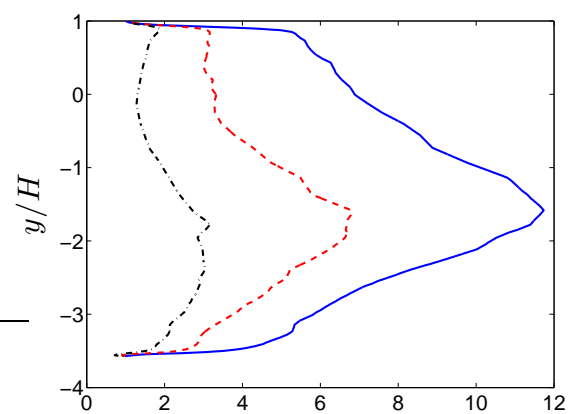

b) $\left\langle\nu_{s g s}\right\rangle / \nu$

Fig. 11. Ratio of SGS to physical viscosity. $N_{x}=256$. Left: $x=-H$; right: $x=20 H$. For legend, see caption in Fig. 2.

$\left\langle\nu_{s g s}\right\rangle / \nu$ is larger at $x=20 H$ than at $x=-H$ which should indicate that the turbulence is worse resolved at the former location than at the latter which is incorrect. Hence, this quantity is not reliable for estimating the resolution.

It is commonly assumed that the SGS dissipation is largest at wavenumbers close to the cut-off. To investigate at which wavenumber the SGS dissipation does takes place, we will investigate the dissipation energy spectra. Since the spanwise coordinate, $z$, is a homogeneous coordinate direction, it is suitable to investigate the energy spectrum of the spanwise component of the SGS dissipation including a spanwise derivative, for example $\partial w^{\prime} / \partial z$. A discrete Fourier transform of $\partial w^{\prime} / \partial z$ is formed as

$$
\begin{aligned}
\hat{D}_{z}\left(k_{z}\right) & =\frac{1}{N_{z}} \sum_{n=1}^{N_{z}} \frac{\partial w^{\prime}(n)}{\partial z} \\
& {\left[\cos \left(\frac{2 \pi(n-1)\left(k_{z}-1\right)}{N_{z}}\right)-\imath \sin \left(\frac{2 \pi(n-1)\left(k_{z}-1\right)}{N_{z}}\right)\right] }
\end{aligned}
$$




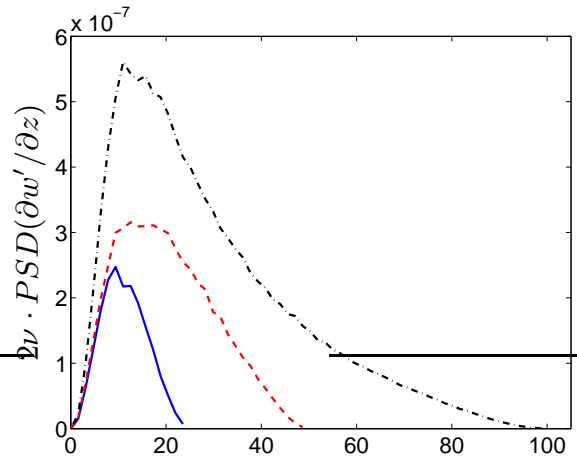

a) $\kappa_{z}=2 \pi\left(k_{z}-1\right) / z_{\max }$

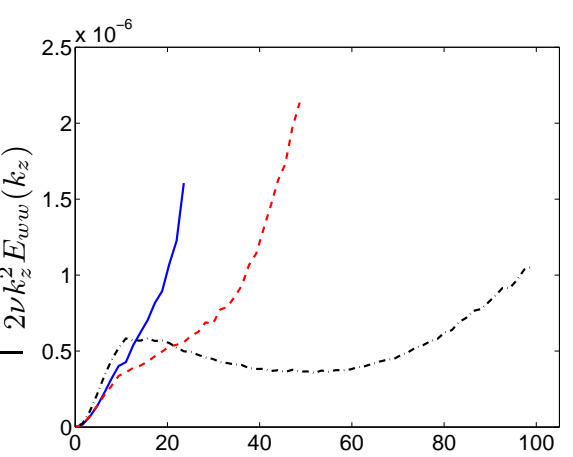

b) $\kappa_{z}=2 \pi\left(k_{z}-1\right) / z_{\max }$

Fig. 12. Exact (left) and approximated (right) dissipation energy spectra of a spanwise component of viscous dissipation versus spanwise wavenumber. For legend, see caption in Fig. 2. $x=-H, y=0.15 H$

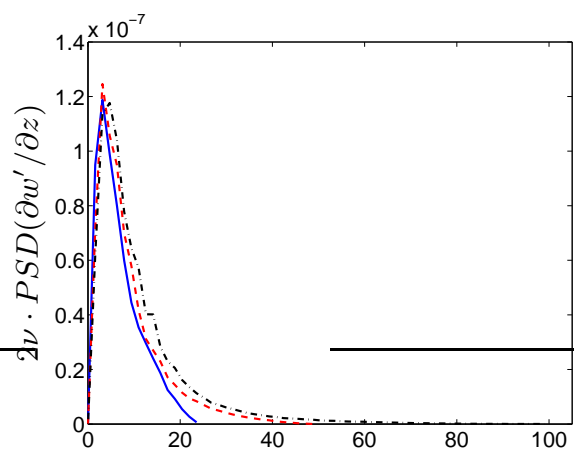

a) $\quad \kappa_{z}=2 \pi\left(k_{z}-1\right) / z_{\max }$

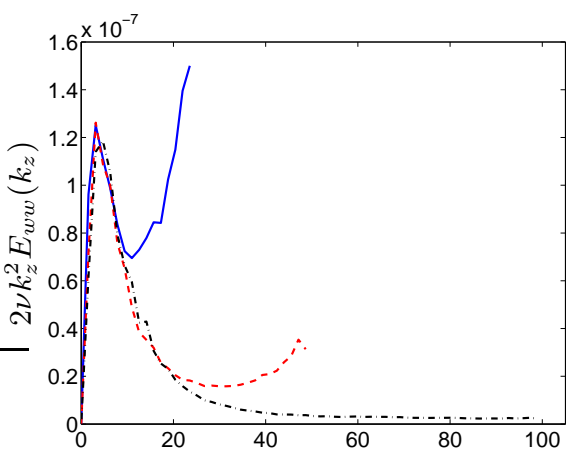

b) $\kappa_{z}=2 \pi\left(k_{z}-1\right) / z_{\max }$

Fig. 13. Exact (left) and approximated (right) dissipation energy spectra of a spanwise component of viscous dissipation versus spanwise wavenumber. For legend, see caption in Fig. 2. $x=20 H, y=-2.9 H$

where $\hat{D}_{z}$ are the complex Fourier coefficients of $\partial w^{\prime} / \partial z$. Then the power spectral density (PSD) of $\partial w^{\prime} / \partial z$, i.e. $\hat{D}_{z} * \hat{D}_{z}^{*}$, where superscript $*$ denotes a complex conjugate, can be formed. The time-averaged value of $\left(\partial w^{\prime} / \partial z\right)^{2}$ can be computed both in physical and wavenumber space, i.e.

$$
\left\langle\left(\frac{\partial w^{\prime}}{\partial z}\right)^{2}\right\rangle=\sum_{k_{z}=1}^{N_{z}}\left\langle\hat{D}_{z} * \hat{D}_{z}^{*}\right\rangle=\sum_{k_{z}=1}^{N_{z}} P S D\left(\frac{\partial w^{\prime}}{\partial z}\right)
$$

The square of the Fourier coefficients, i.e. $\hat{D}_{z} * \hat{D}_{z}^{*}$, is computed, and time and spanwise averaged at run-time in the LES code.

The viscous dissipation corresponding to $\partial w^{\prime} / \partial z$ for $x=-H$ is shown in Fig. 12a. As can be seen, it is largest at surprisingly small wavenumbers. In 


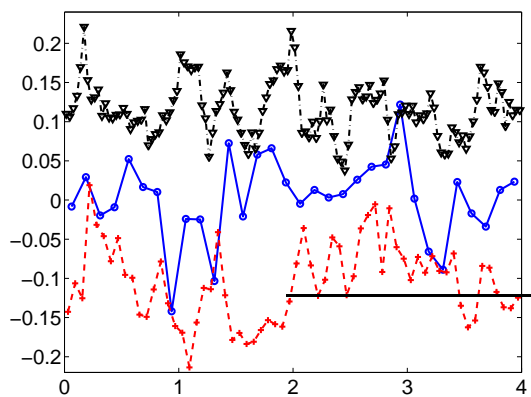

a) $z / H$

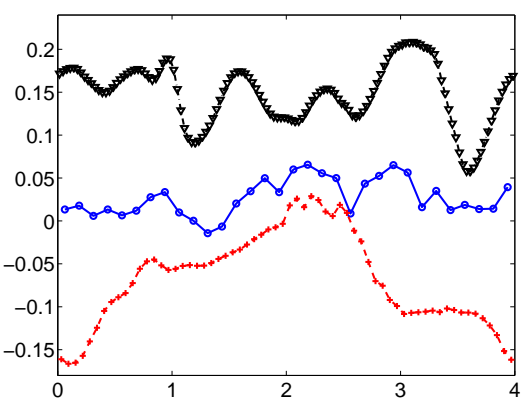

b) $z / H$

Fig. 14. Instantaneous velocity. Left: $x=-H, y=0.15 H$; right: $x=20 H, y=$ $-2.9 H . \_, \circ: N_{k}=32, \bar{w} ;---,+: N_{k}=64, \bar{w}-0.1 ;-\cdots, \nabla: N_{k}=128, \bar{w}+0.13$.

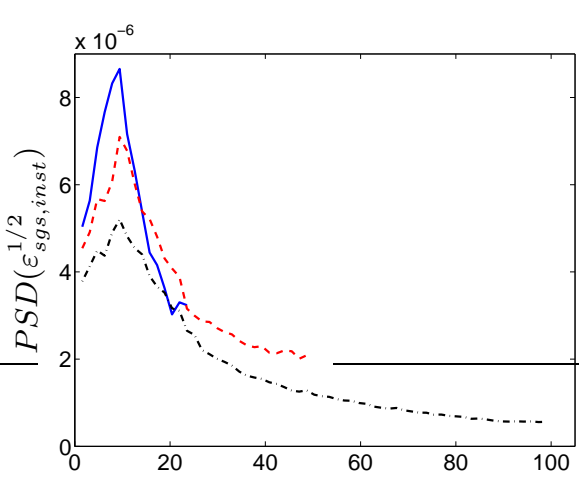

a) $\kappa_{z}=2 \pi\left(k_{z}-1\right) / z_{\max }$

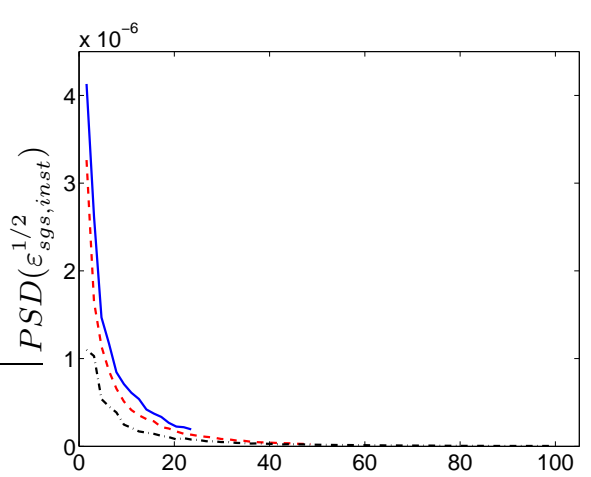

b) $\kappa_{z}=2 \pi\left(k_{z}-1\right) / z_{\max }$

Fig. 15. Exact dissipation energy spectra of the SGS dissipation, versus spanwise wavenumber. Left: $x=-H, y=0.15 H$; right: $x=20 H, y=-2.9 H$. For legend, see caption in Fig. 2.

the inertial subrange with a $\kappa_{z}^{-5 / 3}$ behaviour, the viscous dissipation should - according to Eq. 9 - vary as $\kappa_{z}^{1 / 3}$.

For $x=-H$ the peaks of the spectra occur at $k_{z}=7, k_{z}=9$ and $k_{z}=9$ for the coarse, medium and fine mesh, respectively (the first wavenumber represents the mean, which is equal to zero). This means that derivative $\partial w^{\prime} / \partial z^{\prime}$ is largest for a length scale of $\ell=z_{\max } / 6 \simeq 5$ cells, $z_{\max } / 8=8$ cells and $z_{\max } / 8=16$ cells for the coarse, medium and fine mesh, respectively. In the incipient separation region the derivatives are largest at even larger length scales (Fig. 13a), which is reasonable, since the large scales are much larger and better resolved (see the two-point correlations in Fig. 6); we obtain $\ell=z_{\max } / 3 \simeq 10$ cells, $\ell=z_{\max } / 2=32$ cells and $\ell=z_{\max } / 2=64$ cells for the coarse, medium and fine mesh, respectively.

The spanwise component of the viscous dissipation, $\varepsilon_{w z}$, can also - in theory — be obtained from [9] 


$$
\varepsilon_{w z}=2 \nu\left\langle\left(\frac{\partial w^{\prime}}{\partial z}\right)^{2}\right\rangle=\left.2 \nu \frac{\partial^{2} B_{w w}(\hat{z})}{\partial \hat{z}^{2}}\right|_{\hat{z}=0}=2 \nu \sum_{k_{z}=1}^{N_{z}} \kappa_{z}^{2} E_{w w}\left(k_{z}\right)
$$

where $\kappa_{z}=2 \pi\left(k_{z}-1\right) / z_{\max }$. However, this relation is not satisfied at the discrete level, because derivative $\partial w^{\prime} / \partial z$ cannot be evaluated exactly in a finite-volume approach; the expression in Eq. 9 is based on an exact evaluation of the derivative. The viscous dissipation, $\varepsilon_{w z}$, for $x=-H$ is presented in Fig. 12b. In theory, the spectra presented in Figs. 12a and 12b should be equivalent. However, as can be seen, there is a large discrepancy between the spectra, especially at high wavenumbers. This discrepancy is a measure of the insufficient accuracy of the finite volume method for evaluating $\partial w^{\prime} / \partial z$ at high wavenumbers (small scales).

Figure 13 presents the exact and approximated spectra in the incipient separation region. The picture is much the same: the maximum dissipation takes place at small wavenumbers, and there is a discrepancy between the exact and the approximated spectra, although the difference is smaller than for $x=-H$. The difference is actually very small for the fine mesh $\left(N_{k}=128\right)$, which indicates that the resolution is good (which is correct judging from the two-point correlation). Comparison of the exact and the approximated spectra could be an interesting approach estimating resolution. Unfortunately, it requires a homogeneous flow direction.

To get a more visual picture of how well the spanwise velocity component is resolved, Fig. 14 presents the $w^{\prime}$ velocity at one chosen instant. As can be seen, the coarse grid results are very poorly resolved and the profile exhibits odd-even oscillations. Each peak is resolved by at least two nodes at the medium mesh, but the velocity profile does not look physical. The finest mesh shows a tendency to be well resolved. The situation at $x=20 H$ is completely different. The velocity profiles on both the medium and the fine grid are very well resolved. The $w^{\prime}$ profile obtained with the coarse grid exhibits some odd-even oscillations but its behavior generally seems to be physical. It should be pointed out that the information from instantaneous pictures can be misleading and that it is safer to look at time-averaged quantities.

We present energy spectra of the spanwise derivative of $w^{\prime}$ in Figs. 12 and 13 and we assume that these spectra are representative for the spectra of the SGS dissipation. Let us check this assumption. To analyze the spectra of the total dissipation, a DFT is created by replacing $\partial w^{\prime} / \partial z$ in Eq. 7 with the square-root of the instantaneous dissipation, i.e. $\varepsilon_{\text {sgs,inst }}^{1 / 2}$ (see Eq. 10). Figure 15 presents the energy spectra of the SGS dissipation. The first wavenumber, which corresponds to $\varepsilon_{\text {sgs,mean }}$ (see Eq. 11), is omitted. The peaks are located at approximately the same $\kappa_{z}$ as those in Figs. 12 and 13, but the peaks in the former ones are more dominant $x=-H$. The amplitude in the SGS spectra also differ more for the different grids than those in Figs. 12 and 13 ; this is most likely related to the different SGS viscosities for the different grids, see Fig. 11. 
Contrary to the case in RANS, where the main role of the turbulence viscosity is to act as a diffusion term in the momentum equation, the main objective of the SGS viscosity in LES is to dissipate resolved turbulent kinetic energy, $k_{\text {res }}=0.5\left\langle u_{i}^{\prime} u_{i}^{\prime}\right\rangle$. The contribution of diffusion of the SGS viscosity in LES is usually negligible compared to that of the resolved turbulence. The SGS dissipation term in the $k_{r e s}$ reads [3]

$$
\begin{array}{r}
\varepsilon_{s g s}^{\prime}=\left\langle\left(\tau_{i j, s g s}-\left\langle\tau_{i j, s g s}\right\rangle\right) \frac{\partial u_{i}^{\prime}}{\partial x_{j}}\right\rangle=\left\langle\left(\nu_{s g s} \frac{\partial u_{i}}{\partial x_{j}}\right)^{\prime} \frac{\partial u_{i}^{\prime}}{\partial x_{j}}\right\rangle \\
=\left\langle\nu_{s g s} \frac{\partial \bar{u}_{i}}{\partial x_{j}} \frac{\partial \bar{u}_{i}}{\partial x_{j}}\right\rangle-\left\langle\nu_{s g s} \frac{\partial \bar{u}_{i}}{\partial x_{j}}\right\rangle\left\langle\frac{\partial \bar{u}_{i}}{\partial x_{j}}\right\rangle=\varepsilon_{s g s}-\left\langle\nu_{s g s} \frac{\partial \bar{u}_{i}}{\partial x_{j}}\right\rangle\left\langle\frac{\partial \bar{u}_{i}}{\partial x_{j}}\right\rangle
\end{array}
$$

where the right side on the first line is obtained because the cross-diffusion term has been omitted in the momentum equations (see Eq. 1). The SGS viscosity also appears in the dissipation term in the $K$ equation, see Fig. 16, which reads [3]

$$
\varepsilon_{s g s, \text { mean }}=\left\langle\tau_{i j, s g s}\right\rangle \frac{\partial\left\langle\bar{u}_{i}\right\rangle}{\partial x_{j}}=\left\langle\nu_{s g s} \frac{\partial \bar{u}_{i}}{\partial x_{j}}\right\rangle \frac{\partial\left\langle\bar{u}_{i}\right\rangle}{\partial x_{j}}
$$

which sometimes may be approximated as

$$
\varepsilon_{\text {sgs,mean,approx }}=\left\langle\nu_{\text {sgs }}\right\rangle \frac{\partial\left\langle\bar{u}_{i}\right\rangle}{\partial x_{j}} \frac{\partial\left\langle\bar{u}_{i}\right\rangle}{\partial x_{j}}
$$

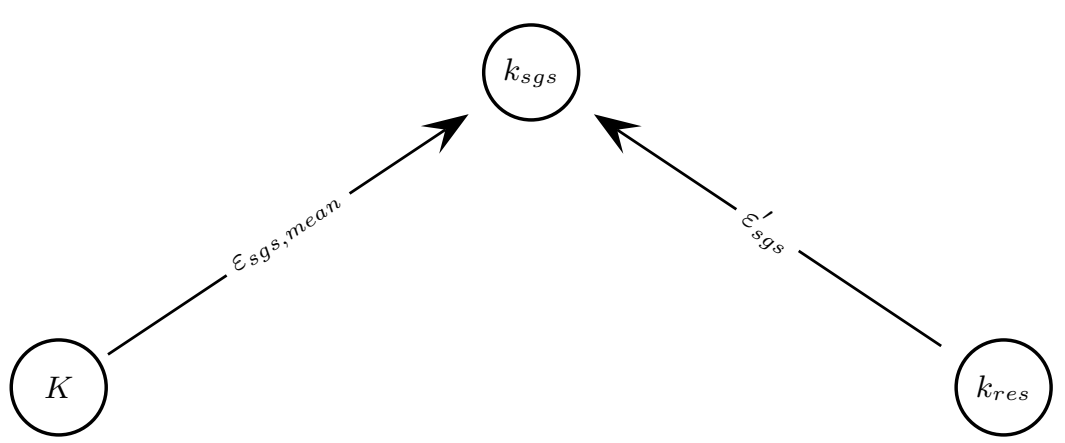

Fig. 16. Transfer of kinetic turbulent energy between time-averaged, resolved and SGS kinetic energy. $K=\frac{1}{2}\left\langle\bar{u}_{i}\right\rangle\left\langle\bar{u}_{i}\right\rangle$ and $k_{\text {res }}=\frac{1}{2}\left\langle u_{i}^{\prime} u_{i}^{\prime}\right\rangle$ denote time-averaged kinetic and resolved turbulent kinetic energy, respectively. $k_{s g s}$ denotes time-averaged SGS kinetic energy. The dissipations, $\varepsilon_{\text {sgs }}^{\prime}$ and $\varepsilon_{s g s, m e a n}$, are defined in Eqs. 10 and 11.

In the same manner, Eq. 10 may sometimes be estimated as

$$
\varepsilon_{\text {sgs,approx }}^{\prime}=\left\langle\nu_{s g s} \frac{\partial \bar{u}_{i}}{\partial x_{j}} \frac{\partial \bar{u}_{i}}{\partial x_{j}}\right\rangle-\left\langle\nu_{s g s}\right\rangle\left\langle\frac{\partial \bar{u}_{i}}{\partial x_{j}}\right\rangle\left\langle\frac{\partial \bar{u}_{i}}{\partial x_{j}}\right\rangle
$$




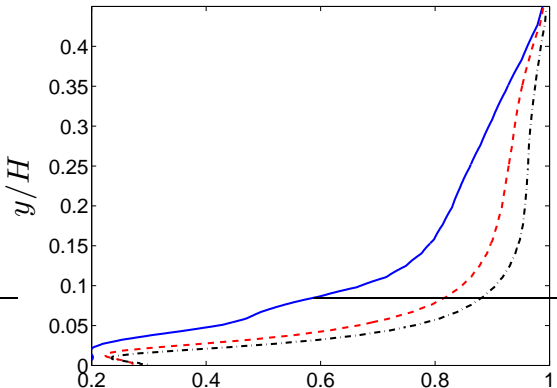

a) $\varepsilon_{s g s}^{\prime} / \varepsilon_{s g s}$

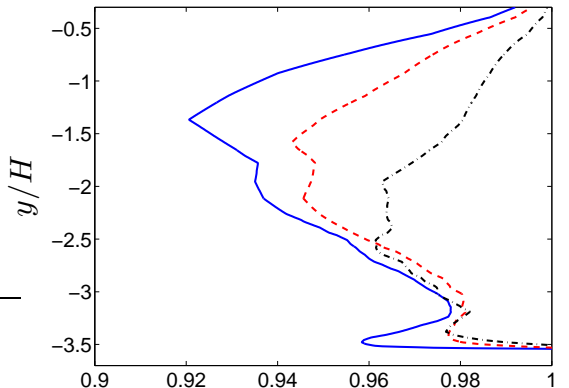

b) $\varepsilon_{s g s}^{\prime} / \varepsilon_{s g s}$

Fig. 17. Ratio of dissipation due to resolved fluctuations, $\varepsilon_{s g s}^{\prime}$, to total SGS dissipation, $\varepsilon_{s g s}=\varepsilon_{s g s, m e a n}+\varepsilon_{\text {sgs }}^{\prime}$. Left: $x=-H$; right: $x=20 H$. For legend, see caption in Fig. 2.

It is found that the approximations, Eqs. 12 and 13, agree within 10\% with their corresponding exact expressions in Eqs. 11 and 10. However, when the turbulent viscosity is large - such as in the URANS region in hybrid LESRANS [6] - the approximation is inaccurate.

The transfer of turbulent kinetic energy is illustrated in Fig. 16. The right part of the figure (i.e. $\varepsilon_{s g s}^{\prime}$ ) vanishes in RANS whereas it dominates in a well-resolved LES. Hence, to estimate how well the turbulence is resolved, it may be interesting to compare $\varepsilon_{s g s}^{\prime}$ and $\varepsilon_{s g s, m e a n}$. This is done in Fig. 17. At $x=-H$, ratio $\varepsilon_{s g s}^{\prime} / \varepsilon_{s g s}$ is larger than $60 \%$, even for the coarse mesh, for $y>0.1$. In the incipient separation region The ratio is larger than $94 \%$ in the incipient separation region for all three meshes. Ratio $\varepsilon_{s g s}^{\prime} / \varepsilon_{s g s}-$ like that of shear stresses (Fig. 10) and viscosities (Fig. 11) - behaves consistently when the resolution is refined, but it is difficult to define a value above which the flow can be defined to be well resolved.

\subsection{Decaying isotropic grid turbulence}

Above it was found that the peak of the SGS dissipation for the diffuser flow is largest at relatively low wavenumbers. This section discussed LES and DNS of decaying isotropic grid turbulence. The objective is to investigate at which wavenumbers the dissipation takes place for DNS and well-resolved LES.

The initial velocity field at $t=0$ is generated from the experimental energy spectrum [2] using the synthetic method in [4]. The domain is a cubic box of side $2 \pi$. Three computations were carried out:

1. fine LES using a Smagorinsky model with $C_{S}=0.1$ on a $128^{3}$ grid;

2. DNS on a $128^{3}$ grid;

3. coarse LES using a Smagorinsky model with $C_{S}=0.1$ on a $64^{3}$ grid. 


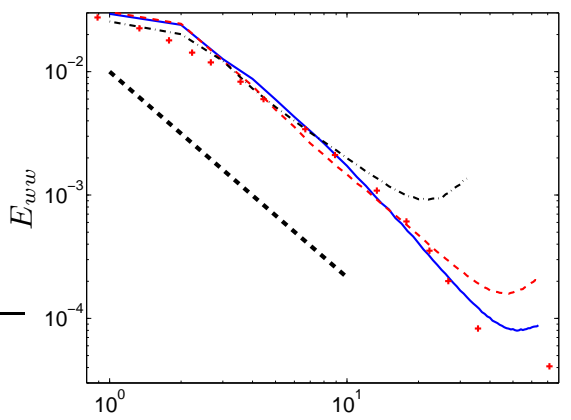

a) $z$

Fig. 18. Decaying grid turbulence. Energy spectra. $t=2$. — : fine LES; - - - : DNS; - - - : coarse LES; Marker: experiments [2]. The thick dashed line shows $-5 / 3$ slope.

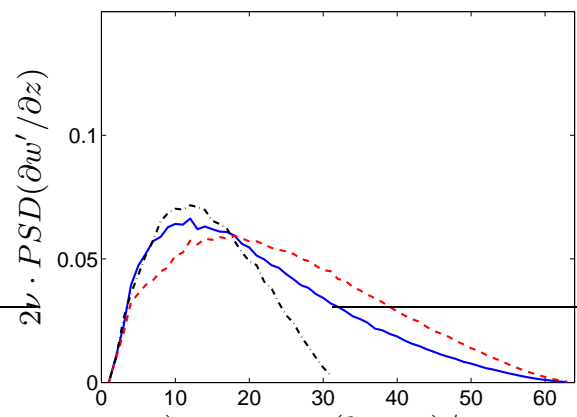

a) $\kappa_{z}=2 \pi\left(k_{z}-1\right) / z_{\max }$

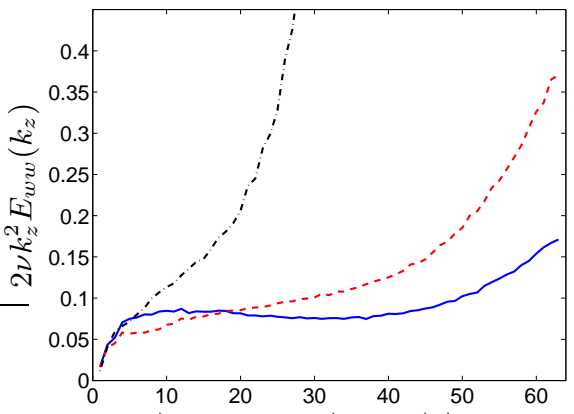

b) $\kappa_{z}=2 \pi\left(k_{z}-1\right) / z_{\max }$

Fig. 19. Decaying grid turbulence. Dissipation energy spectra. $t=2$. Exact (left) and approximated (right). — : fine LES; - - - : DNS; - - - : coarse LES.

Figure 18 presents the one-dimensional energy spectra computed from the two-point correlations. The predicted amplitudes are scaled so that $u_{r m s}^{2}$ agrees with the experimental value; the scaling factors are approximately equal to two. All spectra agree fairly well with the experimental spectrum and they have a $-5 / 3$ decay over a rather large wavenumber range (the fine grid more than a decade). All predicted spectra exhibit a pile-up of energy at the largest wavenumbers. The pile-up is, as expected, smallest for LES on the $128^{3}$ mesh because the fine resolution together with the SGS model helps to dissipate the smallest scales. On the coarse $64^{3}$ grid, the resolution is too coarse and the SGS model does not succeed in introducing sufficient SGS dissipation at the smallest scales to compensate for the poor resolution.

Figure 19a shows that the peak of the fluctuating velocity gradients occurs at wavenumbers $k_{z}=14, k_{z}=14$ and $k_{z}=12$ for the fine LES, the DNS and the coarse LES, respectively. This gives length scales of $\ell=z_{\max } / 13 \simeq 9$ cells, 


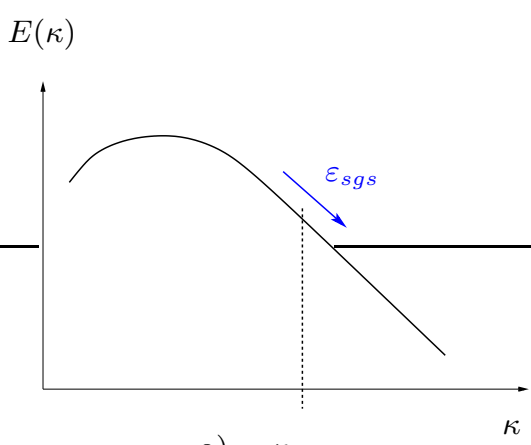

a) $\kappa_{c}$

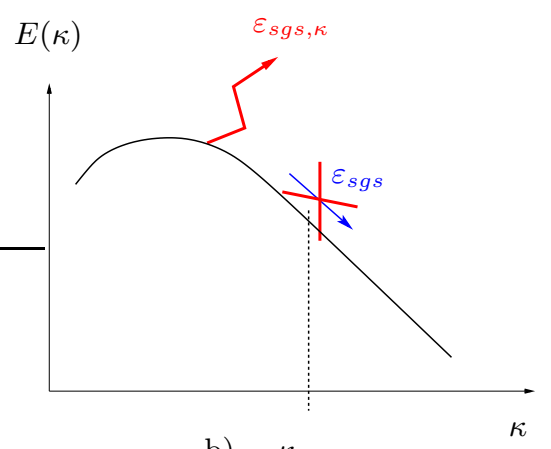

b) $\kappa_{c}$

Fig. 20. Energy spectra. SGS dissipation according to the idealized picture (a) and SGS dissipation according to the present work (b).

$z_{\max } / 13 \simeq 9$ cells and $\ell=z_{\max } / 11 \simeq 6$ cells, respectively. As expected, we find that the SGS model on both the the fine and the coarse mesh moves the location of the peak in the fluctuating velocity gradients towards slightly smaller wavenumbers compared to the DNS. Moreover, it can be seen that the LES on the fine and coarse grids gives the same location of the peak, indicating that - except for the smallest scales - the coarse resolution is sufficient.

The approximated spectra in Fig. 19b all exhibit a maximum at the highest wavenumbers. This is in accordance with the pile-up of energy in the energy spectra, confirming that the smallest scales are not well resolved on any grid.

In conclusion, it is found that also for DNS and well-resolved LES, the dissipation takes place at surprisingly small wavenumbers.

\section{Concluding remarks}

Various ways of estimating resolution in recirculating flow have been considered.

It is concluded that the most useful quantity for estimating resolution are two-point correlations. They show by how many cells the largest scales are resolved. It is then up to the CFD user to judge how many cells are required; at least eight to ten cells seems to be reasonable.

The energy spectra do not give reliable information about the resolution.

The ratio of the SGS to the molecular viscosity, $\left\langle\nu_{s g s}\right\rangle / \nu$, and the ratio of the SGS to the resolved shear stress behave consistently upon grid refinement, i.e. they decrease. However, the ratio $\left\langle\nu_{s g s}\right\rangle / \nu$ indicates incorrectly that the turbulence is better resolved in the attached flow region of the diffuser than in the incipient flow region. Hence, the ratio $\left\langle\nu_{s g s}\right\rangle / \nu$ is not a reliable quantity for estimating the resolution.

Energy spectra of resolved fluctuating gradients and SGS dissipation give information about the wavenumbers at which the SGS dissipation takes place. It is commonly assumed that the SGS dissipation occurs close to the cut-off 
wavenumber, see Fig. 20a. The present study (as was found also for channel flow in [6]), however, show that this idealized picture is not true. Instead the SGS dissipation takes place at rather low wavenumbers, see Fig. 20b. Note that this conclusion does not apply for spectral methods for which the relation Eq. 9 is valid; in these methods $\varepsilon \propto \kappa^{1 / 3}$ applies in the inertial region, see for example [11]. In the present study the length scales related to the peaks of the SGS dissipation correspond to approximately 10 cells (spanwise direction) in attached flow and much more in the incipient separation region. This was confirmed in DNS and well-resolved LES of decaying grid turbulence. The disadvantage of these quantities is that they can only be used in flows that possess a homogeneous direction, which is seldom the case in real flows.

Time-averaged velocity gradients and the resolved fluctuating velocity gradient both contribute to SGS dissipation, denoted by $\varepsilon_{s g s, m e a n}$ and $\varepsilon_{\text {sgs }}^{\prime}$, respectively. The latter is zero in RANS, whereas it dominates in well-resolved LES. Hence, the ratio $\varepsilon_{s g s}^{\prime} /\left(\varepsilon_{\text {sgs }}^{\prime}+\varepsilon_{\text {sgs,mean }}\right)$ may be useful in evaluating resolution. It is found that this ratio is very large in recirculating flow (more than $94 \%$ ), revealing good resolution. However, as mentioned with respect to the ratio of the shear stresses and and the viscosities, it is difficult to give any recommendations for threshold values.

\section{Acknowledgments}

The financial support of SNIC (Swedish National Infrastructure for Computing) for computer time at C3SE (Chalmers Center for Computational Science and Engineering) is gratefully acknowledged.

\section{References}

1. Buice, C., Eaton, J.: Experimental investigation of flow through an asymmetric plane diffuser. Report No. TSD-107, Thermosciences Division, Department of Mechanical Engineering, Stanford University, Stanford, California 94305 (1997)

2. Comte-Bellot, G., Corrsin, S.: Simple Eularian time correlation of full- and narrow-band velocity signals in grid-generated "isotropic" turbulence. Journal of Fluid Mechanics 48(2), 273-337 (1971)

3. Davidson, L.: Transport equations in incompressible URANS and LES. Report 2006/01, Div. of Fluid Dynamics, Dept. of Applied Mechanics, Chalmers University of Technology, Göteborg, Sweden (2006)

4. Davidson, L.: Using isotropic synthetic fluctuations as inlet boundary conditions for unsteady simulations. Advances and Applications in Fluid Mechanics 1(1), $1-35$ (2007)

5. Davidson, L.: A dissipative scale-similarity model. In: DLES7: Direct and LargeEddy Simulations 7. 8-10 September, Trieste, Italy (2008)

6. Davidson, L.: Large eddy simulations: how to evaluate resolution. International Journal of Heat and Fluid Flow 30(5), 1016-1025 (2009) 
7. Davidson, L., Peng, S.H.: Hybrid LES-RANS: A one-equation SGS model combined with a $k-\omega$ model for predicting recirculating flows. International Journal for Numerical Methods in Fluids 43, 1003-1018 (2003)

8. Emvin, P.: The full multigrid method applied to turbulent flow in ventilated enclosures using structured and unstructured grids. Ph.D. thesis, Dept. of Thermo and Fluid Dynamics, Chalmers University of Technology, Göteborg (1997)

9. Hinze, J.: Turbulence, 2nd edn. McGraw-Hill, New York (1975)

10. Klein, M., Sadiki, A., Janicka, J.: A digital filter based generation of inflow data for spatially developing direct numerical or large eddy simulations. Journal of Computational Physics 186(2), 652-665 (2003)

11. Schlatter, P., Stolz, S., Kleiser, L.: Evaluation of high-pass filtered eddy-viscosity models for large-eddy simulation of turbulent flows. Journal of Turbulence 6(5), $1-21(2005)$

12. Wu, X., Schlüter, J., Moin, P., Pitsch, H., Iaccorino, G., Ham, F.: Computational study on the internal layer in a diffuser. Journal of Fluid Mechanics 550, 391412 (2006) 